\title{
Aggregation of retail stores
}

\author{
Pablo Jensen* (a,b), Jean Boisson (a) and Hernán Larralde (c) \\ (a) LPMCN-CNRS, Université Lyon-1, 69622 Villeurbanne Cedex, FRANCE \\ (b) LET-CNRS, Université Lyon-2, 69007 Lyon, FRANCE \\ (c) Centro de Ciencias Físicas, UNAM, Cuernavaca Mor. Apdo. Postal 48-3, C.P. 62251, \\ Cuernavaca, Morelos, MEXICO
}

\begin{abstract}
We propose a simple model to understand the economic factors that induce aggregation of some businesses over small geographical regions. The model incorporates price competition with neighboring stores, transportation costs and the satisfaction probability of finding the desired product. We show that aggregation is more likely for stores selling expensive products and/or stores carrying only a fraction of the business variety. We illustrate our model with empirical data collected in the city of Lyon.
\end{abstract}




\section{INTRODUCTION}

A rather trivial observation is that some businesses tend to aggregate over small geographical regions. In some streets there are nothing but jewelers while other streets are solely dedicated to electronic equipment. Indeed there are many examples of trades that tend to "cluster": trendy clothes shops, art dealers, secondhand book stores, used cars dealers, furniture stores, etc. On the other hand there are trades like barbers, butchers or bakers, which in general do not agglomerate, but rather tend to be uniformly distributed over the entire urban zone.

Under the assumption that the principal consideration for a businessman to choose the location of his business is to maximize his profits, it seems intriguing that businesses in the same trade would choose to be neighbors. After all, proximity forces them to share the market and to compete amongst each other. This is more intriguing given the fact that other

trades driven by the same consideration of maximum profit have a markedly distinct behavior, choosing locations as far as possible from the competition. From a physics perspective, it is tempting to identify this situation with some sort of condensation phenomenon in which interacting particles equilibrate into a condensate or gaseous phase, which optimizes (in this case minimizes) a free energy, depending, say, on the temperature of the system. However, it is not clear how stores "interact", nor the sense in which the distribution of stores can be considered an equilibrium state. Furthermore, the optimization of the profit, which we assume drives the decision of where to locate a business, is an individual decision which generally does not lead to a globally optimized state as in thermodynamics. Thus, before trying to approach this problem through an analogy with a physical system, we need to understand the economic factors that give rise to these phenomena. In this respect there is, in fact, a long history on the study of spatial competition in economics, starting with the model by Harold Hotelling (the original reference is [1], for more recent analysis, see [2-5], for empirical studies, see [6]).

In this work we present a simple model incorporating some of the essential economic 
determinants involved in consumer decision and we try to assess the effect these factors have in the spatial distribution of stores. We discuss the principal features of our model and present analytical and numerical results obtained for a 2-dimensional town.

\section{CLUSTERING COEFFICIENT}

Before going into the details of the model we need to determine what we are looking for. In particular, as the main phenomenon we are trying to understand is the aggregation of stores, we need a measure of this quantity to characterize the outcome of our model. We begin by considering a geographically extended system (which can represent a city, a country, a road, a beach, etc.) which we divide into small regions. Those regions containing stores will be possible shopping sites (we will refer to them just as "sites"). The idea is that the businesses within a site are close enough for a customer to visit them all when searching for an item. In a very important sense we are assuming that when customers decide where to make a purchase, they actually choose among the various sites, rather than among individual stores, though, of course, some sites may contain a single store. The actual size of the regions that constitute a site is relatively arbitrary and depends on the scale of description. Thus, sites may be as large as cities if the complete system is a large country, or a few blocks if the system corresponds to a city. In any case, the size of a site should be such that customers will consider a trip to any of the stores at a given site as being of essentially the same length, and thus, of the same cost. For the business owners the presence of other stores within the distance which defines the site will force them to compete for their share of the market.

We will characterize the tendency to aggregate in a system in which there are $N_{\text {stores }}$ stores distributed among $N_{\text {sites }}$, sites through the following clustering coefficients:

The clustering coefficient $C$ defined as :

$$
C=1-N_{\text {sites }} / N_{\text {stores }}
$$

Which has the following behavior: $\mathrm{C}=0$ when all the stores are isolated and $C \simeq 1$ when all 
the stores aggregate in a single site. This coefficient does not represent a complete description of the store distribution among the sites (for example, 30 stores can be distributed among 3 sites as $1,1,28$ or $10,10,10$, both leading to $C=0.9)$.

A different measure of the store agglomeration is given by the mean site size is defined as:

$$
N_{m}=\frac{\sum_{\text {sites } i} N_{i}^{2}}{\sum_{\text {sites } i} N_{i}}=\frac{\sum_{\text {sites } i} N_{i}^{2}}{N_{\text {stores }}}
$$

which is the mean number of stores per site. This quantity allows to discriminate among the preceding examples, since it equals 26.2 and 10 respectively.

Table I gives an idea of how these coefficients reflect the degree of clustering of different businesses in the city of Lyon. The difference in the values of the clustering coefficients results from the difference in the spatial distribution which can be appreciated in the figures showing the locations of the businesses on the map of Lyon (Figure 1).

\section{ECONOMIC INGREDIENTS OF THE MODEL}

We will focus on single unspecified trades, under the simplifying assumption that the interaction between different trades is not a relevant factor for the geographical clustering of businesses [7]. We begin by characterizing the sites $s$, which are points (representing small regions) in a geographically extended system at each of which there are $N_{s} \geq 1$ businesses. These $N_{s}$ are the central quantities of this work: if profit is optimized when $N_{s}>1$ there will be clustering of businesses. This behavior will be reflected by the clustering coefficients defined previously.

In our model, sites have four characteristics : a position, the number of businesses $N_{s}$, the variety of items it offers and the price of the products sold there. Clearly, the variety is a non decreasing function of $N_{s}$. We also consider that, due to competition, having various stores at a given site might have an effect on the price offered at that site. Barring the

possibility of cartels, we expect that the price $p_{s}=p\left(N_{s}\right)$ will be a non growing function of 
$N_{s}$, the number of stores at the site $s$.

Now, to proceed, we need to characterize the buyers that form the market for which each site competes. We begin by assuming that each buyer $j$ can assign to each site $s$ a perceived probability of satisfying his need $V_{s}(j)$. There may be many factors affecting $V_{s}(j)$, including advertisement, but whatever the peculiarities of buyer $j$, it is again certain that $V_{s}(j)$ should be a non decreasing function of the variety of site $s$, and therefore a non decreasing function of the number of stores at the site. Another element that affects the decision of which site to visit is the cost of getting there: if we denote by $\mathbf{r}_{j}$ and $\mathbf{r}_{s}$ the positions of buyer $j$ and site $s$ respectively, we denote this cost as $C\left(\mathbf{r}_{j}, \mathbf{r}_{s}\right)$. Finally, we assume that each consumer has a maximum amount he is willing to pay for the desired item, which we denote as $R(j)$ $(R(j)$ can also be thought as the "utility" of this good to consumer $j)$.

The choice of which site consumer $j$ will patronize is achieved by maximizing a criterium function $K\left(R(j), V_{s}(j), C\left(\mathbf{r}_{j}, \mathbf{r}_{s}\right), p_{s}\right)$ over all sites, where in general this function may vary from one buyer to another. However, once again, it is clear that $K\left(R(j), V_{s}(j), C\left(\mathbf{r}_{j}, \mathbf{r}_{s}\right), p_{s}\right)$, which we will call the customer's surplus, should be a decreasing function of $C\left(\mathbf{r}_{j}, \mathbf{r}_{s}\right)$ and of $p_{s}$; and a growing function of $V_{s}(j)$. We define $K$ so that a consumer buys only if its maximum $K$ is positive.

To see how this works consider an artificially simplified market, for example soft drinks. Since most convenience stores offer the complete available variety of soft drinks at basically the same price, the perceived probability of satisfying your crave for a drink is essentially 1 at any of these stores (sites) (of course, real convenience stores sell more products than soft drinks, but for the sake of argument, let us assume that soft drinks constitute their trade). Then, for each buyer, $K$ will be maximized at the site for which $C\left(\mathbf{r}_{j}, \mathbf{r}_{s}\right)$ is minimum. That is, at the store of easiest (cheapest) access, which is of course what one expects. Thus, for the market of soft drinks a convenience store caters to the customers contained in a region, namely the region for which site $s$ is the most convenient choice. If a second shop sets up next to the first, the site now has 2 stores but the perceived probability of satisfaction of the customers, being already optimal, will not change. If there is no markdown in prices due to 
competition, the site with two stores will cater to the same customers as when there was a single store, and both stores will end up dividing the original profits.

Our model is based on the assumption that the new stores will appear in the system at the locations that maximize their profit. Thus, one of the factors that is partly responsible for the geographical distribution of stores is the capture of market share. The share of an aggregated site is large because a geographical grouping of many shops increases the consumer's expected satisfaction probability of finding the desired product at that "shopping site" and, perhaps, at a lower price. The model also includes factors that push against an aggregated distribution (or, in the economic terminology, that push for differentiation of the products on the spatial dimension) such as lowering of the consumer transportation cost and the decrease in profits due to price competition between neighboring shops $[3,5]$.

\section{WRITING DOWN THE EQUATIONS}

\section{A. Characterization of consumers and sites}

The scenario we set up in the previous section is too vague to lead to concrete predictions. In this section we choose specific functional forms for the various quantities involved in the system. Perforce our choices are relatively arbitrary: we use simple functions which comply with the monotonicity conditions mentioned above and which interpolate between intuitively acceptable cases.

First we introduce the customer's surplus function, which we will assume to be given by the total expected utility of shopping at a site. That is, the criterium on which consumer $j$ decides whether or not to shop at site $s$ will be given by:

$$
K_{j s} \equiv K\left(R(j), V_{s}(j), C\left(\mathbf{r}_{j}, \mathbf{r}_{s}\right), p_{s}\right)=R(j) V_{s}(j)-C\left(\mathbf{r}_{j}, \mathbf{r}_{s}\right)-p_{s}
$$

Note that since we are assuming that consumers never buy at sites for which $K$ is negative,

the above form discards all sites for which the transportation costs plus the retail price exceeds $R$. 
Now, for the probability of satisfaction of a given site $s$ with $N_{s}$ stores we will assume that

$$
V_{s}(j)=1-\exp \left(-N_{s} / Z\right)
$$

for all customers $j$. The value of $Z$ will vary from trade to trade depending on the complete variety offered in the market. Thus, $Z$ will be small (smaller than 1 ) for trades in which stores typically carry the whole variety in the market (newspaper stores, convenience stores, boulangeries ...). Conversely, $Z$ will be large in trades in which there is a high variety and there is little overlap between the items offered at the various stores (used book sellers, motorbike stores, art galleries ...).

Next, we will assume that the cost of transportation is proportional to the geometrical distance between the customer and the site being considered:

$$
C\left(\mathbf{r}_{j}, \mathbf{r}_{s}\right)=a\left|\mathbf{r}_{j}-\mathbf{r}_{s}\right| \equiv a d_{s j}
$$

where $a$ is the cost per unit distance.

For the price offered by the site, we take

$$
p(s)=p_{0}+b / N_{s}^{\alpha}
$$

where $p_{0}$ is the cost price of the product, $b$ is the maximum store margin for that product and $\alpha$ is an exponent reflecting the strength of price competition among stores at the same site. If $\alpha=0$ there is no competition (all the stores, isolated or aggregated, offer the same price). This particular expression for the price competition is suggested by the "circular city model" [3,8,9] and a study of a basic oligopoly model [4]. Finally, as a first approximation, we will assume $R$ to be the same for all customers and that customers are uniformly distributed throughout the city.

Then, in principle, consumer $j$ will buy at the location $s$ which maximizes his surplus, i.e. the location for which $K_{j s}$ is maximum (and positive), where this quantity is explicitly given by

$$
K_{j s}=R\left(1-\exp \left(-N_{s} / Z\right)\right)-a d_{j s}-\left(p_{0}+b / N_{s}^{\alpha}\right)
$$




\section{B. Demand of a site and profit}

The actual choice of business location falls on the business owners. We are assuming that these will choose the site that maximize their profit and this profit will depend on the number of consumers that can be expected to attend the chosen site once the new business is in place, as well as on the price that the local competition forces on the businesses at that site.

\section{Non interacting sites}

To estimate the expected profit for a business, we begin by introducing the demand $D_{s}^{\prime}$ of site $s$ as the number of consumers that choose to attend this site, i.e.

$$
D_{s}^{\prime}=\sum_{j} \delta_{j s}
$$

where $\delta_{j s}=1$ if $K_{j s}>K_{j s^{\prime}}$ for every site $s^{\prime} \neq s$ and $\delta_{j s}=0$ otherwise.

As mentioned above, we have assumed that if $\max _{s} K_{j^{\prime} s}<0$ the consumer $j^{\prime}$ chooses not to buy; thus, we define the fraction of the market covered by the stores as the proportion of costumers who effectively buy something. This will allow to distinguish between the situations of partial market coverage versus complete market coverage and will allow us to define a kind of "range of interaction" among sites. Specifically, consider a system containing a single site $s$ with $N_{s}$ stores in an unbounded domain. We define the range of this site, denoted by $l_{s}$ as the distance at which

$$
R\left(1-\exp \left(-N_{s} / Z\right)\right)-a l_{s}-\left(p_{0}+b / N_{s}^{\alpha}\right)=0 .
$$

That is, $l_{s}$ is the distance to the boundary beyond which customers will not patronize the site because it would result in a negative surplus. Clearly, the region within this boundary contains the demand of site $s$. This range is a growing function of $R, \alpha$ and $N_{s}$; and a decreasing function of $a, p_{0}$ and $b$. The significance of these ranges of demand is also clear: sites located in such a way that the regions contained within their ranges of demand do 
not overlap, do not compete with one another for customers and the increase in the market attending one site does not translate into a loss of market for the other sites. In this sense, these sites do not "interact". Such a situation can only happen at low market coverages, where still a large fraction of the customers are not willing to acquire the article offered at any of the sites. Then, if it is profitable to do so, at low enough market coverages new sites can appear to cater to the unattended customers without interacting with the previous sites on the system. If these new sites appear randomly in the system, as long as their demand ranges still do not overlap, the problem can be mapped directly to a sequential adsorption problem with exclusion, for which a jamming transition occurs [10]. After this transition point, every new site will have a demand range that overlaps with the demand range of previous sites and interaction between different sites begins to play a rôle in the system. We will return to this point later.

The caveat of profitability mentioned in the last paragraph is important. Indeed, the range of demand as defined above is a growing function of the number of stores at a site, however the profit accrued by the individual stores at the site is not necessarily so. We will assume that the actual profit $\left(\operatorname{Prof}\left(N_{s}\right)_{s}\right)$ which each store stands to gain at site $s$ to be given by:

$$
\operatorname{Prof}\left(N_{s}\right)_{s}=\left(p_{s}\left(N_{s}\right)-p_{0}\right) D_{s}^{\prime}\left(N_{s}\right) / N_{s}
$$

This is an important quantity in the model as it is the quantity upon which businesses decide where to locate. It is again an undoubtedly oversimplified assumption. The idea is that the addition of a store to a site modifies the demand for that site but the eventual profits are the result of complicated maneuvering by all the stores, matching supplies and competing in price. The above expression assumes that the outcome will result in an equal share of the site's demand among the stores, and that all the stores at the site sell at a uniform competition adjusted price, achieving the same profit. 


\section{Interacting sites}

Of course, in any finite system, as more and more sites appear, their ranges will always end up overlapping and the simple analysis presented above breaks down.

To analyze what happens in general when the market coverage is high and ranges of demand of the distinct sites do overlap we must recur to numerical simulations. However, in this situation the expressions for $K_{j s}$ and $D_{s}^{\prime}$ used in the previous discussion lead to relatively unrealistically sharp discontinuities in consumer preferences, as already noted by Hotelling [1]. Therefore, we have taken a less sharp expression for the demand, assuming that there exists some unknown random factors that "smoothen" the consumer's choice among similar stores. A simple way for smoothing the demand of each site is to use the well-known (in economics) "logit" function [4]:

$$
D_{s}=\sum_{j} \sigma_{j s}
$$

where

$$
\sigma_{j s}=\frac{\exp \left(K_{j s} / T\right)}{\sum_{s^{\prime}} \exp \left(K_{j s^{\prime}} / T\right)}
$$

where only the sites for which $K_{j^{\prime} s}>0$ are considered. In this expression $T$ is a parameter which defines how sharply consumers discern between the expected surpluses offered by each site. As $T \rightarrow \infty$ consumers do not discriminate between eligible sites, whereas if $T \rightarrow 0$ we recover the sharp condition of Eq.(8). Furthermore, in systems containing single or noninteracting sites customers will have at most one site to choose from, so $D_{s}=D_{s}^{\prime}$ for any $T$. In what follows, the profit will be calculated in terms of this demand function through the expression

$$
\operatorname{Prof}\left(N_{s}\right)_{s}=\left(p_{s}\left(N_{s}\right)-p_{0}\right) D_{s}\left(N_{s}\right) / N_{s}
$$




\section{CUALITATIVE BEHAVIOR: NON INTERACTING SITES}

For the simple case in which the market coverage is extremely low, the system will contain a set of noninteracting or isolated sites. An estimate of the profit of each store as a function of $N_{s}$ in this situation can be obtained by noting that the demand is proportional to the area within the range of demand of a site. Approximating this area by a circle, and given the explicit functional forms we have assumed, the profit will be given by

$$
\operatorname{Prof}\left(N_{s}\right) \approx \rho \frac{\pi b}{N_{s}^{\alpha+1}}\left[R\left(1-e^{-N_{s} / Z}\right)-p_{0}-\frac{b}{N_{s}^{\alpha}}\right]^{2}
$$

where $\rho$ is the number of consumers per unit area.

As mentioned above, in this situation, the profit of each store in a site is not necessarily a monotonous function of the number $N_{s}$. Indeed, depending on the value of the parameters characterizing the system, the profit can grow as $N_{s}$ increases, up to a maximum and then decrease to zero. Or else, the profit may be a purely decreasing function of $N_{s}$ (see figure 2),

depending on the variety coefficient $Z$, which is the major determinant of store aggregation. Only values of $\mathrm{Z}$ higher than 1 lead to aggregation. It should be noted, however, that $N_{s}^{\text {opt }}$ does not correspond to the site size that is actually reached in a simulation. Stores will continue to aggregate at a site beyond $N_{s}^{o p t}$ as long as it is more profitable to do so than to establish a new site. Take for example the case $Z=5$ in Figure $2 \mathrm{~b} . N_{s}^{\text {opt }}$ equals 8 , but the $9^{\text {th }}$ store still finds more profitable to locate within the site than alone where it would get the profit corresponding to $N_{s}=1$. This is true until the $124^{\text {th }}$ enters the site (not shown in Figure 2b). On the other hand, if $Z<1$, stores will find it more profitable to locate far from the existing stores to avoid the overlap of their range of demand with those of the previous stores as long as this is possible. This situation is akin to a short range repulsion between stores. 


\section{APPLICATION TO A SQUARE CITY : NUMERICAL RESULTS}

When the ranges of demand overlap, the determination of the expected profit to be made by new stores is not as simple as in the preceding cases and we must resort to numerical simulations.

While most extensions of the Hotelling model have considered the situation in which stores are distributed over a one dimensional space (for an exception dealing with two stores, see Ref. [11]), in this work we concentrate on the distribution of stores in a 2-dimensional square city of area $L^{2}$ with a uniform density of consumers. In our numerical simulations we choose $b=R / 10$, i.e. a maximum store margin of $10 \%, a=1 / L$ to normalize the distances and $p_{0}=0$ (except in section VIC 3).

The process we want to reproduce is simple enough: we assume that businesses are able to estimate the expected profit for each location, and choose the location in which they expect to earn the most. Depending on the existing configuration and the value of the parameters, the chosen site can be one in which other stores are already present, leading to store aggregation, or an isolated location. It is, of course, the outcome of these decisions that will lead to the final distribution of businesses.

The algorithm for simulating this process is very simple: for each set of parameters, after putting by hand the first store in the center of the town, we repeat the following steps until the desired number of stores has been attained:

(1) scan all the possible sites in the town (i.e. $L^{2}$ possible sites) and calculate the profit to be earned at each location, given the location of the stores already present.

(2) Put a new store at the location which maximizes the profit, and start again (1).

We stress that in this work we do not consider business mobility. Thus once a store chooses its location, it remains there forever. This is an extreme case, applicable to businesses which have high costs of relocation. Also, the profits of some stores diminish drastically as more stores enter the system. In real economic systems, stores earning too little eventually go bankrupt and disappear. Furthermore, systems should saturate once they reach the situation 
in which all stores present have profits above the bankruptcy level but every possible new entry leads to profits below that level. In this work we will assume that stores survive even when perceiving extremely small profits, so there will be no bankruptcies nor saturation effects. However, we note that very small profits only arise when the parameter $T$ in the "logit" function appearing in Eq. 11 is very small, i.e. consumers buy at the best location exclusively.

\section{A. Repulsive Regime}

We begin considering trades for which the profits expected in noninteracting sites are a purely decreasing function of $N_{s}$. We start with the case in which the range of interaction is small compared to the size of the city, and new stores faced with several equivalent possibilities (in the low coverage regime) choose among these possibilities randomly.

First we consider the case in which $Z<<1$ and market coverage is low, so there is no competition. This situation is repulsive, as discussed above, since the profits are decreasing functions of $N_{s}$. Thus we expect the system to pass from a random sequential adsorption scenario at the beginning, where each new entry finds enough unattended market to choose sites in which it does not interact with the previous sites, to a cluttered configuration, in which each new entry searches the site at which it can bite off the most market from the previous stores. In figure 3 we show the profit of each new store as it enters the system, which stays constant for the first 8 stores, and then decays, when the market coverage is high enough. The same behavior is observed for any value of $\alpha$, since inclusion of lower price due to price competition does not affect demand in the system and all sites continue having only one store each.

We now turn to the case in which the range of demand is of the order of the size of the city. This gives rise to strong boundary effects: the range of demand of stores located near the edge of the city extend beyond the city limits, so these stores would have less market than locations near the center of the city. Thus even a single store would be "pushed" 
away from the boundaries and if the range is of the order of the size of the city, will try to locate close to the city center. As we illustrate in a numerical example below, this boundary effect will also lead to nonuniform store distributions, with many stores near the center, that resemble store aggregation even when we are dealing with sites in the repulsive regime.

\section{B. Clustering as a function of $R$}

Fig. 4 shows the clustering coefficient obtained for $\alpha=1, Z=1, L=50$ and 70 stores (and, as always, $b=R / 10$ ). There exists a clear trend towards agglomeration when the utility $R$ increases. However, there are jumps in the clustering coefficient, which we now discuss in more detail.

For $R<5$, the shops are totally disaggregated to avoid decreasing the retail price due to competition. However, they are not homogeneously distributed over the city (Fig. 5, for $R=3$ ), since there exists a preference for a central position, which minimizes the distance to consumer's locations. This situation suddenly changes around $R=5$ because for this system, this value of $R$ represents a threshold at which the 66th store gets a higher profit when aggregating with the first one (at the very center of the town) instead of finding some other (isolated) place. Fig. 6 helps to understand why this is so. It shows the (decreasing) profits for the stores as they enter the market and also the (hypothetical) profits when aggregation is forbidden. As long as the best choice is to remain isolated, the two curves are of course identical, and follow a simple law : the profit for the $n^{\text {th }}$ single store entering the

town is given by $P_{1}=b D_{1} \simeq \frac{R}{10 n}$, showing that all stores roughly share the total demand. However, when it becomes more profitable for an entering store to aggregate, the two curves split, and at first sight, profits seem smaller for the "free" case than for the isolated case. A close inspection of the numerical results shows that the first store that decides to aggregate effectively finds there a higher profit opportunity. This is because the demand of this 2-store site is much larger than that of single sites, in fact 4 times higher to compensate for the loss of profit from price competition. This higher demand, in turn, comes from the high $\mathrm{R}$ value, 
which allows the satisfaction factor to dominate all the other terms in Eq. 7. This is why there is no clustering for smaller values of $\mathrm{R}$ in Fig. 4. The point is that once this 2-store site is created, it modifies the whole market, "gulping" a substantial share of the demand, so that all the subsequent stores find no better solution than to aggregate too. In some sense the "selfish" decision of the 66th store put the system in an inconvenient situation in which the maximum profits of subsequent stores are lower than they would have been had all the stores remained isolated. As $\mathrm{R}$ increases, this first aggregation event takes place sooner, leading to a higher clustering coefficient.

\section{Effects of the other parameters}

We now examine the effects of the different parameters in more detail.

\section{Effect of variety}

We start with the influence of $Z$ : small (smaller than 1) for trades in which stores typically carry the whole variety in the market and large in trades in which there is a high variety.

Our results are summarized in Fig. 7. For $Z=5$, clustering is always larger than for $Z=1$ for the following reason. The satisfaction factor is low $(1-\exp (-1 / 5)=.181$ for $N=1$ and 0.33 for $N=2$ ), to be compared to 0.632 for $N=1$ and $Z=1$ ). Therefore, stores have to cluster in order to generate enough demand, otherwise $K_{i s}$ is lower than 0 for virtually every consumer (for example, for $R=1$, the market coverage in our model city is of only $2 \%$ for 2 stores at the center, but increases to $25 \%$ for 3 stores). For small $Z$, only single stores exist, since they carry the whole variety $\left(V_{s} \simeq 1\right.$, see Eq. 4$)$. Then, as

discussed previously, aggregation of stores only leads to profit sharing, without increasing the demand significantly. 
Fig. 8 shows the influence of $\alpha$ for $Z=1$ and $Z=5$. Stores are more agglomerated for $\alpha=0$ than for $\alpha=1$ as the outcome of the competition between two opposite effects. The $\alpha$ value changes the price dependence on the number of clustered stores, strong for $\alpha=1$ and unexistent for $\alpha=0$. However, the effect of the price on the store profit is not direct, but the combination of two factors. A lower price $(\alpha=1)$ obviously decreases the profit per sale, but increases the demand. It is the competition between these two factors that determines the clustering effect. In our model (Fig. 8), the effect of dismissing price competition $(\alpha=0)$ favors clustering, i.e. the increase of price always exceeds the losses due to the demand factor, at least for high enough $R$ values $(R>4)$.

That this should be the case is again easy to see in the case of non interacting sites. Indeed, from Eq. 14, it is straight forward to verify that the profit is a decreasing function of $\alpha$. Then, since the expected profit when $N_{s}=1$ is the same independently of $\alpha$, when the parameters are such as to favor aggregation, the maximum profit for $\alpha>0$ will be achieved at a lower number of stores than when $\alpha=0$. Similarly, the number of stores beyond which profits fall below those of a single isolated store is smaller for $\alpha>0$. However, it is by no means obvious how these effects carry over to the case of interacting sites.

\section{Effect of $p_{0}$ and $T$}

For completeness, we have also examined the effects of the other parameters, $p_{0}$ and $T$, and also the effect of a sharp consumer choice, without the logit smoothing. The effect of the first one is trivial, since it only induces a difference with $p_{0}=0$ when $R<p_{0}$, as shown in Fig. 9.

The "temperature" $T$ has subtler effects. Figure 10 shows that there are two regimes : for $R<1$, all the curves are similar, while for $R>1$ the temperature matters and the curves split. The first regime can be easily understood by noting that if $K_{j s} / T<<1$, the 
exponentials in Eq. 12 are essentially 1 and T disappears from the demand calculation. To understand the second regime, let us note that, compared to the "reference" case $(\mathrm{T}=1)$, decreasing the temperature leads to a discrimination of the "best" site, while a higher T leads to a uniform demand among all sites. Therefore, when $\mathrm{T}$ is large, competing stores tend to isolate, since their demand is essentially unaffected, but their prices (and profits) are much higher. Instead, when consumers pay attention to prices, the competition between aggregation and isolation is subtler : for small $R$, aggregation increases the demand (via a larger market coverage) efficiently enough to compensate for the price competition, and stores aggregate. When $R$ increases, agglomeration is not so interesting and a compromise is found which depends on the precise value of $R$.

The effects of a sharp consumer choice (i.e. $T=0$ or, equivalently, demand given by Eq. 8) are shown in Fig. 11. When consumers choose to buy exclusively at the "best" store (that with the highest $\mathrm{K}$ ), as in the previous cases, the agglomeration is always higher for $\alpha=0$ or $/$ and $Z=5$.

\section{DISCUSSION}

Let us put our results in the perspective of previous analysis of the store aggregation phenomena.

Here, the main economical force that leads to aggregation is the capture of market share. The share of an aggregated site is large because a geographical grouping of many shops increases the consumer probability of finding the desired product in that region, and reduces the prices. Alternatively, factors that push for a homogeneous distribution (i.e. a differentiation of the products on the spatial dimension) are the decrease of the consumer transportation cost and the avoidance of price competition between shops.

The common view in marketing texts is that differentiation is important to soften price competition. However, there are several economical forces that push against too much differentiation, as summarized by Tirole [3] : 
(1) Be where the demand is : this pushes all the stores to be located near the center of the town, or near "strategic" places where the demand is concentrated, for example restaurants near working places for lunch time, etc. The "center" effect is recovered in our model (see Fig. 5), but since our city is homogeneous, we cannot reproduce the "strategic" effect.

(2) Positive externalities between firms : if there are common installations (for example fishermen along the same harbor) or a source of raw materials. Alternatively, consumers may encourage firms to gather, because this lowers their research cost. One can argue that aggregation should be an interesting option when consumers visit more than one shop before buying a given product. This point is particularly relevant for non standard products, for variety seeking would be pointless if products sold by neighboring stores would be perfect substitutes. This point is clearly shown in Ref. [5] : "In some activities, the basic reason for the agglomerative tendency is that the outputs [...] differ in such manifold and changing ways that they cannot be satisfactorily compared by the buyer without actual inspection. [Take the example of] a man or woman buying a car or a new hat $[. .$.$] : the buyer does$ not know exactly what will be purchased. The items cannot be adequately described in a catalog, and it would be much too expensive and time consuming for the producers to supply each prospective buyer with a full set of samples. Under these circumstances, the "demand" is not so much demand for specific items as it is demand for a varied display of products; and the wider the variety presented at a particular location, the more demand that location will attract. It is clear that the activity that is presenting the displays will tend to adopt a clustered pattern, with its units positively attracting one another. A newcomer to the cluster may even be welcomed, because that seller will enrich the variety and draw still more demand to the location." Our model clearly illustrates this second effect, most clearly through the variety factor $Z$. The higher $Z$ values (see paragraph VIC 1 ) show that demand for variety effectively leads to clustering.

(3) Absence of price competition : in some cases, for legal or technical reasons, prices are exogeneously fixed. Therefore, there is no direct push for differentiation. In Hotelling's model, this induces aggregation at the center of the one-dimensional "town" for two firms. 
When there are more than two firms, the analysis is more complex and has been carried out only in one-dimensional spaces [4]. Here, absence of price competition means $\alpha=0$, and we have seen that this value always lead to higher aggregation than $\alpha=1$.

It can be shown [4] that for a 1D space, $n$ firms charging equal prices and competing over locations (with a demand obtained through a logit function), there are different equilibria depending on the value of T/a. Roughly, for low values there is no equilibrium, for intermediate values there are equilibria with stores located outside the center of the interval, while aggregation of stores at the middle of the "town" is the only equilibrium for high values. This result is interpreted as "minimal differentiation" on the spatial dimension provided there is enough differentiation in the characteristics (of the goods sold) space, represented by the $\mathrm{T}$ value appearing in the logit, or when consumers put more weight on nongeographical dimensions (less sensitive to price, i.e. to transportation in this model without price differences among stores).

Thus, we believe that our rather simplified model captures most of the essential economic features of the system. We have also shown that by simply changing the parameters of the model we can reproduce qualitatively the behavior of different trades.

Furthermore, our approach highlights the fact that the distribution of stores is the result of strategic decisions of business owners as a response to strategic decisions of the customers. Thus, two different models are actually involved: one for strategy of the businessmen (maximum immediate profit in this case) and one for the strategy of the customers (maximum surplus, though tempered by a logit function). From this setup, an effective interaction arises between stores trying to attain maximum profits given the location of the stores that preceded them.

\section{PERSPECTIVES}

From the point of view of modelling economic systems many improvements could be included to the model presented in this work. Such improvements would basically confer 
more realism and flexibility to the model but would probably not give rise to qualitatively new behaviors. In particular, one could introduce a "better" description of the interactions amongst neighboring sites, which is essential for price competition and satisfaction. This could be done by substituting the dependence on $N_{s}$ in our formulas by an "effective" $N_{\text {eff }}$ which would take into account the presence of stores at the neighboring sites. Such a modification would correspond to spatially extending the effects of competition and variety beyond the simplified pointlike sites we have used in this work.

One important ingredient which for the sake of simplicity we have left out is real price competition within and between sites. In respect to competition within sites, it is interesting to note that if the stores at an isolated site were able to set the price of maximum profit, the price offered by the site would be a growing function of the number of stores at the site. This is true under the assumption that all the stores sell at the same price, however, this cartel practice would be unstable due to price competition within the site. The actual equilibrium price, if it exists at all, seems hard to find even for this simple case.

In regards to price competition between sites, we would require to consider the possibility that once a store chooses the most profitable site, it may do so at the expense of the profits at other sites. These affected sites might then react by lowering the price to recover, at least partially, some of their lost profit, affecting other sites which react similarly. Once again, what the equilibrium price distribution is, if any, very difficult to determine.

A different extension could be to consider the possibility of relocation of stores after all of them have entered the town, sequentially maximizing their profits, or during this process. However, these relocations may come at a cost to the stores, which will hinder their mobility even if a better site exists. Still some of them will prefer to move to find better profits and it would be interesting to find out whether equilibrium configurations exist and whether they can be reached. Along the same lines, it should also be interesting to refine the description of the economics of store life: for example to calculate the annual profit and delete those stores who do not make enough profits to survive.

Finally, the implicit hypothesis that consumers are omniscient, knowing all stores location 
and prices is not realistic. In the spirit of the "agent based" modelling [12], one could start with consumers that only know their closest store and then randomly visit the town to learn the different prices and varieties offered by alternative stores, with the possibility of sharing this information with their neighbors. This would allow to study how a store deciding to change its prices influences the behavior of the consumers, and possibly the other stores as well.

From the physics perspective the opposite is probably true. It would be satisfactory to find a minimal realistic model based on the economic ingredients of the system, that could reproduce the observed phenomena. One venue could be to propose interaction forces among stores that mimic the customer mediated interactions in this system. This would be an important first step in the direction of casting this problem into a nonequilibrium many body physical system, however, it does require a good understanding of these economic interactions.

A deep problem, intrinsic to many economic models, is that most of the parameters that characterize the system are not readily accesible to direct measurement. Thus, another interesting line of inquiry would be to develop methods to estimate these parameters a priori in real economic systems. Otherwise, these models are explanatory but lack useful predictive capabilities.

* e-mail address : jensen@lpmcn.univ-lyon1.fr

We acknowledge fruitful discussions with Alain Bonnafous, Jean-Louis Rouhtier (LET) and Robert Axtell (Washington). 


\section{REFERENCES}

[1] H. Hotelling, Economic Journal 3941 (1929)

[2] J. Hinloopen, C. van Marrewijk, International Journal of Industrial Organization 17 735 (1999)

[3] J. Tirole, The theory of Industrial Organization (MIT Press, Cambridge, 1988)

[4] Anderson, M. Fujita and J. F. Thisse, Discrete Choice Theory of Product Differentiation (MIT Press, Cambridge, 1992)

[5] Edgar M. Hoover and Frank Giarratani, An Introduction to Regional Economics (Alfred A. Knopf, 1984)(available on the Web via The Web Book of Regional Science (www.rri.wvu.edu/regscweb.htm))

[6] P. J. Davies, "Spatial competition in retail markets : movie theaters" (2002) Ph D dissertation, available on the Web at http://web.mit.edu/pjdavis/www/; J. S. Netz and B. A. Taylor, The Review of Economics and Statistics 84162 (2002)

[7] This is an approximation, since shopping centers and downtown businesses take advantage of the demand generated by stores of different trades to increase their own demand. However, the its effect on aggregation of similar businesses is not clear.

[8] S. Salop, Bell Journal of Economics 10141 (1979)

[9] M. Fujita and J. F. Thisse, Economics of Agglomeration: Cities, Industrial Location, and Regional Growth(Cambridge University Press, Cambridge, 2002)

[10] J. W. Evans, Rev. Mod. Phys. 651281 (1993)

[11] E.C.H. Veendorp and Anjum Majeed, Regional Science and Urban Economics 2575 (1995)

[12] See the special issue of the Proceedings of the National Academy of Sciences 99 (3) (14 
May 2002); Joshua M. Epstein, Robert L. Axtell, Growing Artificial Societies: Social Science from the Bottom Up (Brookings Institution Press, Washington) (1996)

Table I Clustering coefficients observed in Lyon for several businesses. The coefficients have been calculated by defining stores grouped in a "site" as stores closer than $l_{\text {ave }} / 3$, where $l_{\text {ave }}$ is the average distance between this kind of stores in the whole town (note that by transitivity, two stores farther away than $l_{\text {ave }} / 3$ can be grouped in the same site if intermediate stores connect them). We then calculate the clustering coefficients as in Eqs.

1 and 2 .

$\begin{array}{lll}\text { Business } & C & N_{m} \\ \text { motorbikes } & 0.45 & 5.6 \\ \text { banks } & 0.44 & 3.3 \\ \text { groceries } & 0.37 & 2.5 \\ \text { hairdressers } & 0.28 & 1.8 \\ \text { laundries } & 0.21 & 1.5 \\ \text { drugstores } & 0.12 & 1.28 \\ \text { savings banks } & 0.046 & 1.08\end{array}$




\section{FIGURES}

(a)

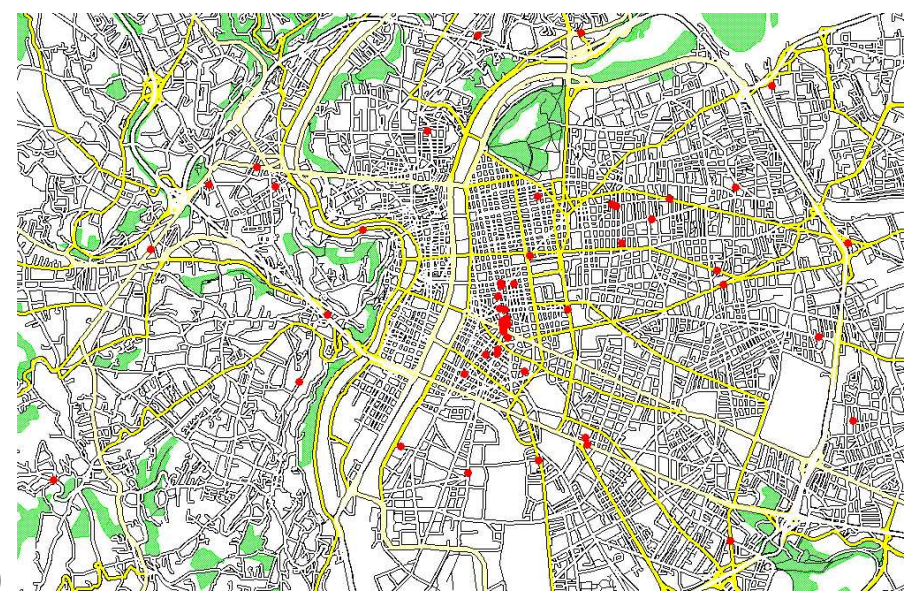

(b)

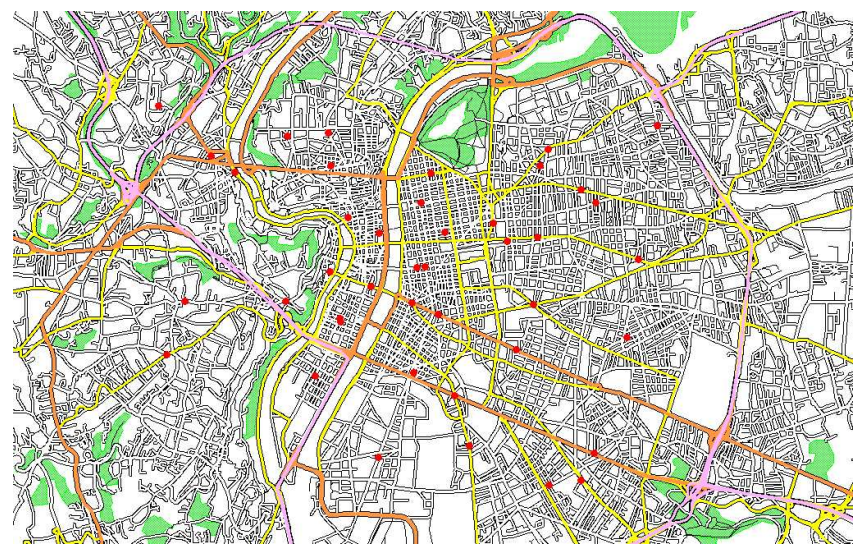

FIG. 1. Location of motorbike sellers (a) and savings banks (b) in Lyon (big dots, 1996 data).

We thank Jean-Louis Routhier (LET-CNRS) for help with this spatial information.

(a)

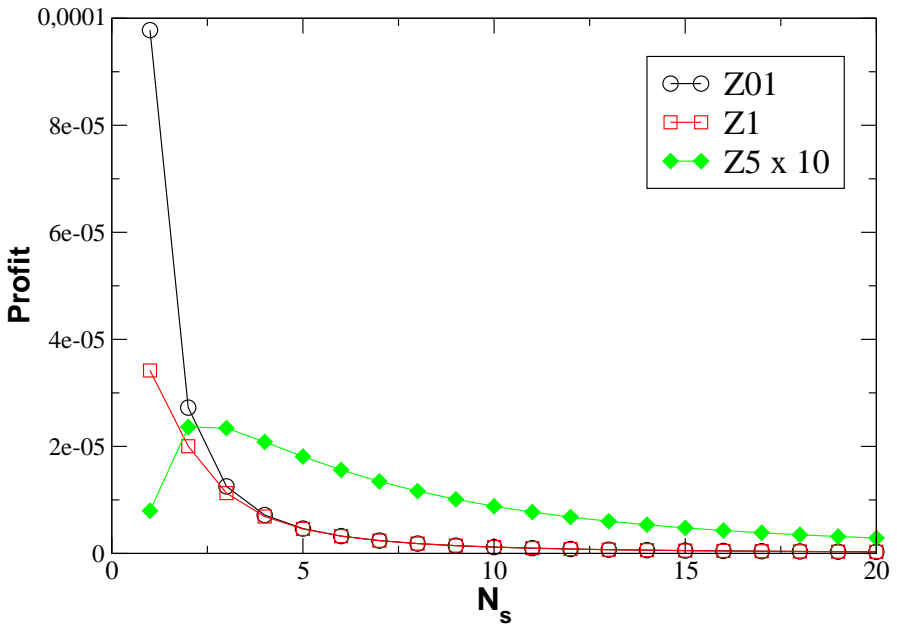

(b)

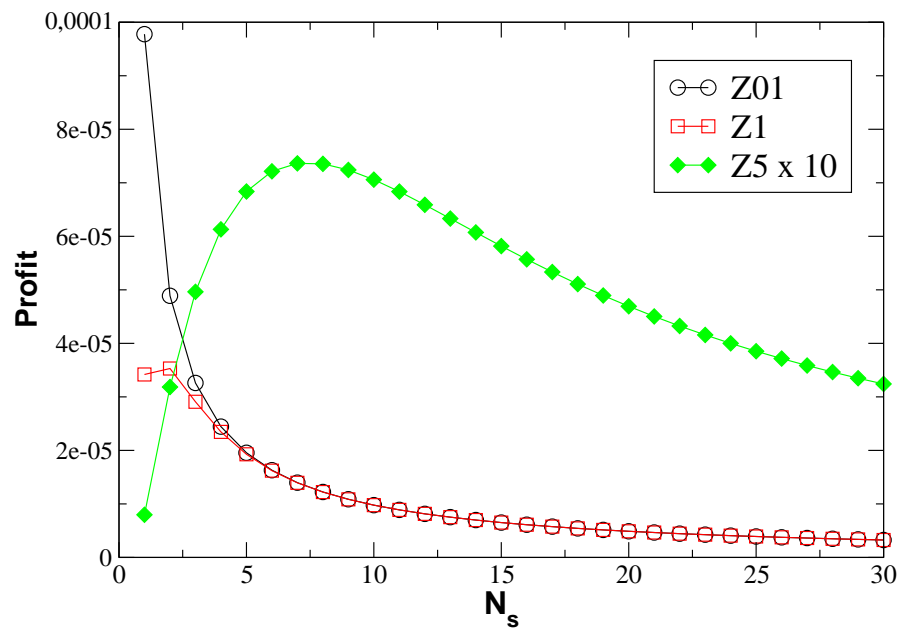

FIG. 2. Profit of a store in an isolated site containing $N_{s}$ stores : (a) $\alpha=1$; (b) $\alpha=0$. The curves refer to $R=1, L=50$ but different values of $Z$ and as indicated in the inserts. 


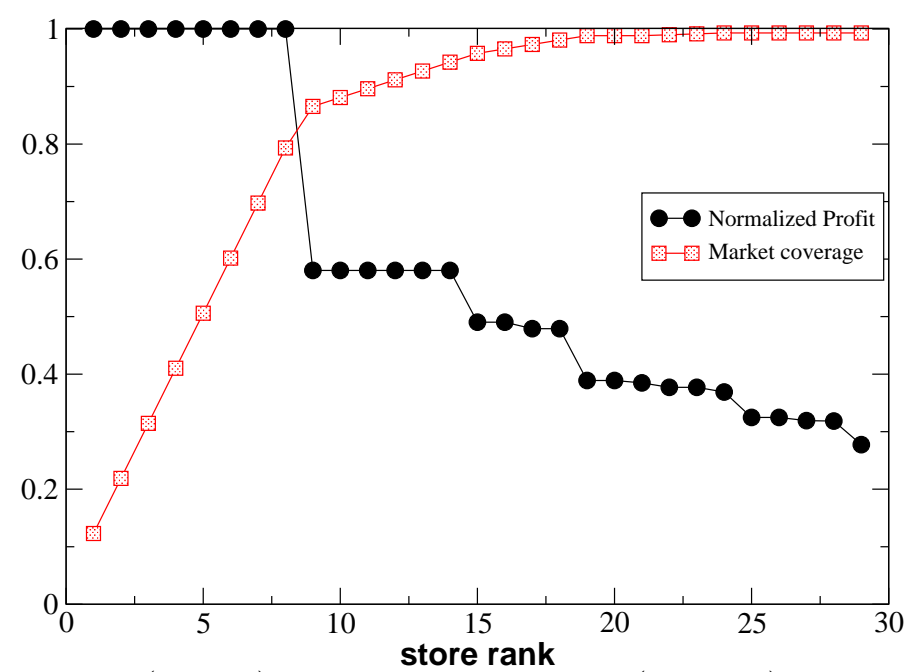

FIG. 3. Normalized profit (circles) and market coverage (squares), as stores enter the city. The profit is normalized to the profit of the first store. The curve has been obtained for $R=0.2, \alpha=0$, $Z=0.1, L=50$ and $N_{\text {stores }}=30$

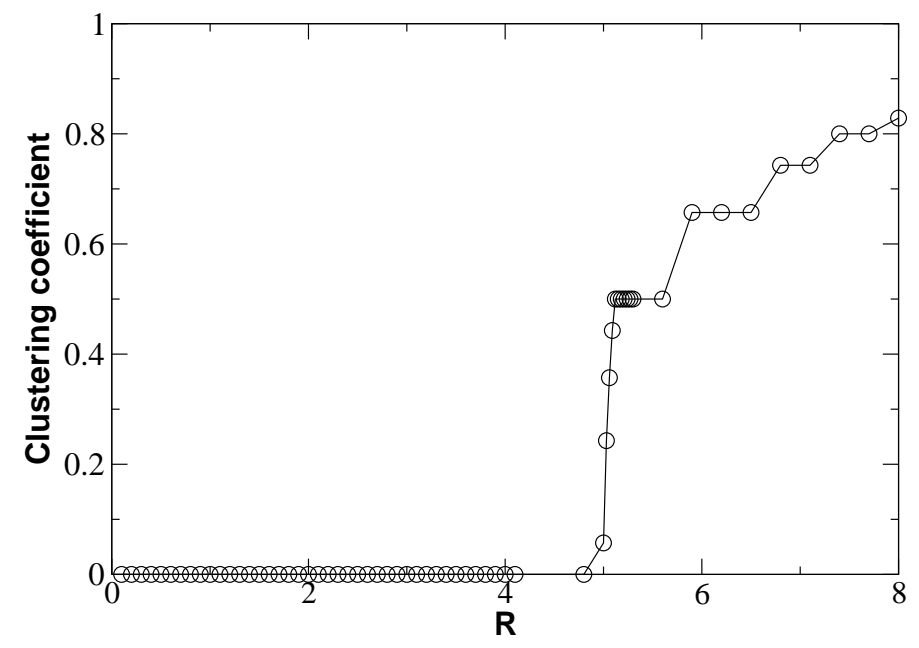

FIG. 4. Clustering coefficient obtained for $\alpha=1, Z=1, L=50$ and $N_{\text {stores }}=70$ 


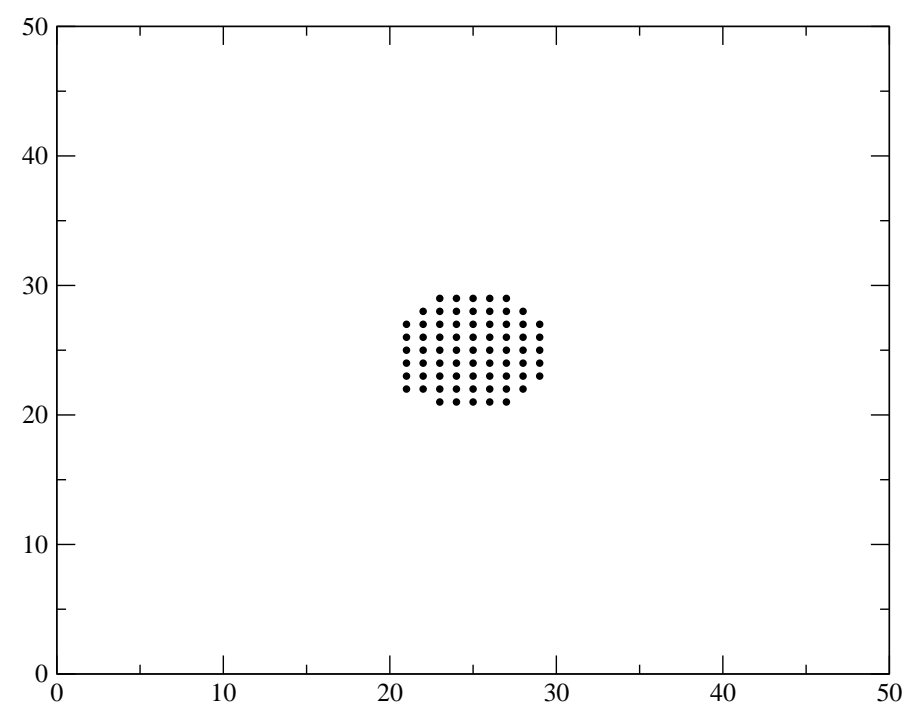

FIG. 5. Location of the (isolated) stores for $R=3, \alpha=1, Z=1, L=50$ and $N_{\text {stores }}=70$

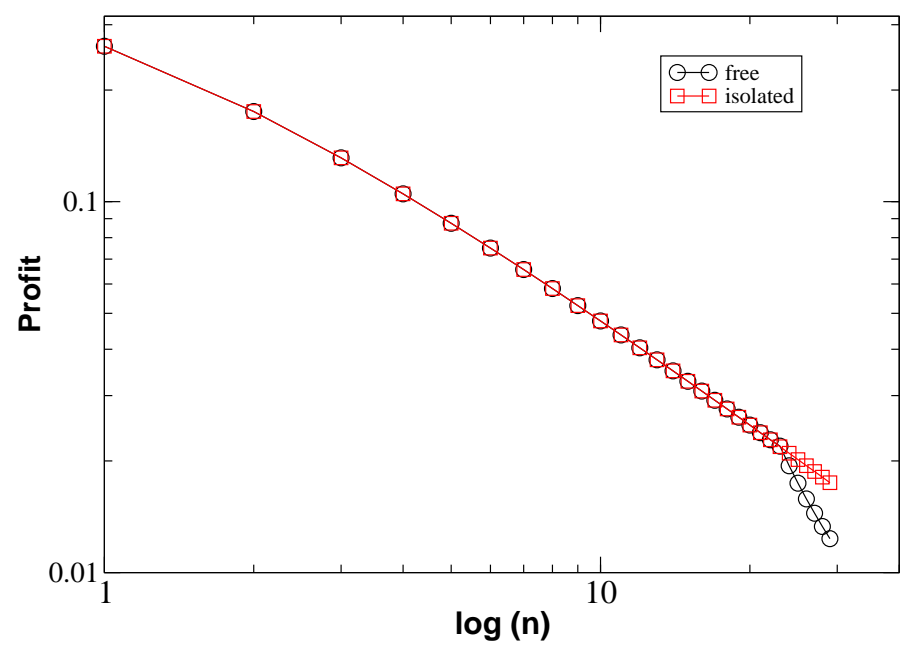

FIG. 6. Profits of stores as they enter the market choosing the most profitable location (circles) and profits when aggregation is forbidden (squares). $R=5.2, \alpha=1, Z=1, L=50$ and $N_{\text {stores }}=30$ 

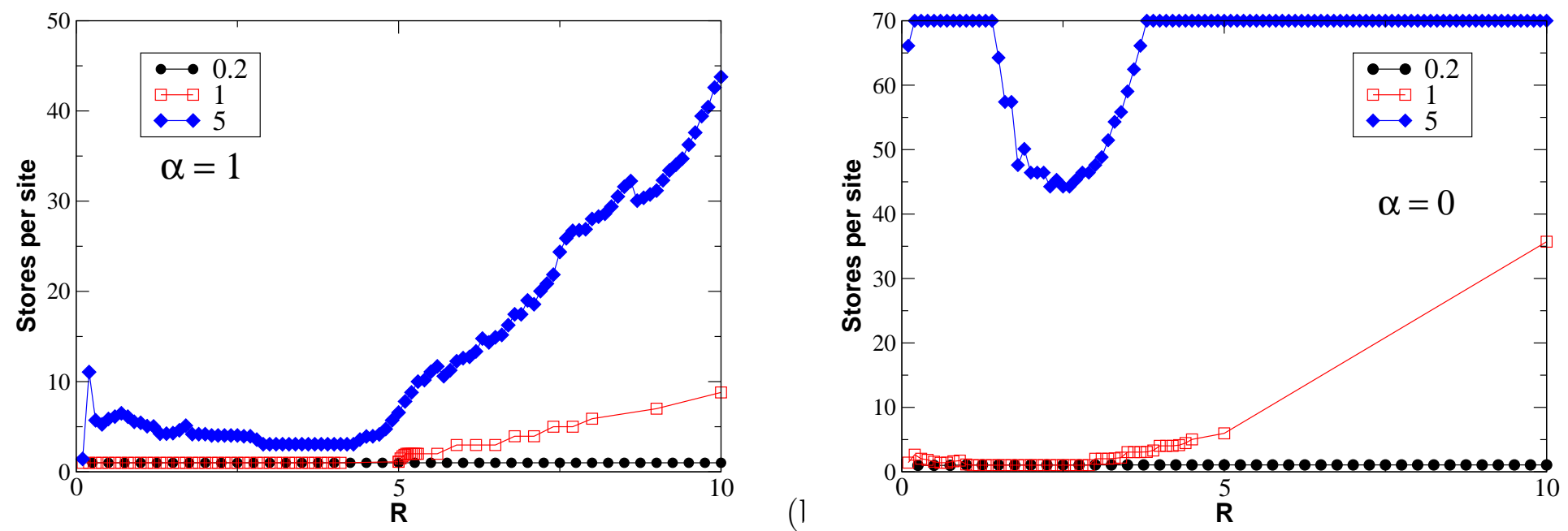

FIG. 7. Mean site size obtained for different values of $Z$ (shown in the inset) and different values of $\alpha$ ( 1 for (a) and 0 for (b)). Other parameters are fixed : $T=1, p_{0}=0, L=50$ and $N_{\text {stores }}=70$.

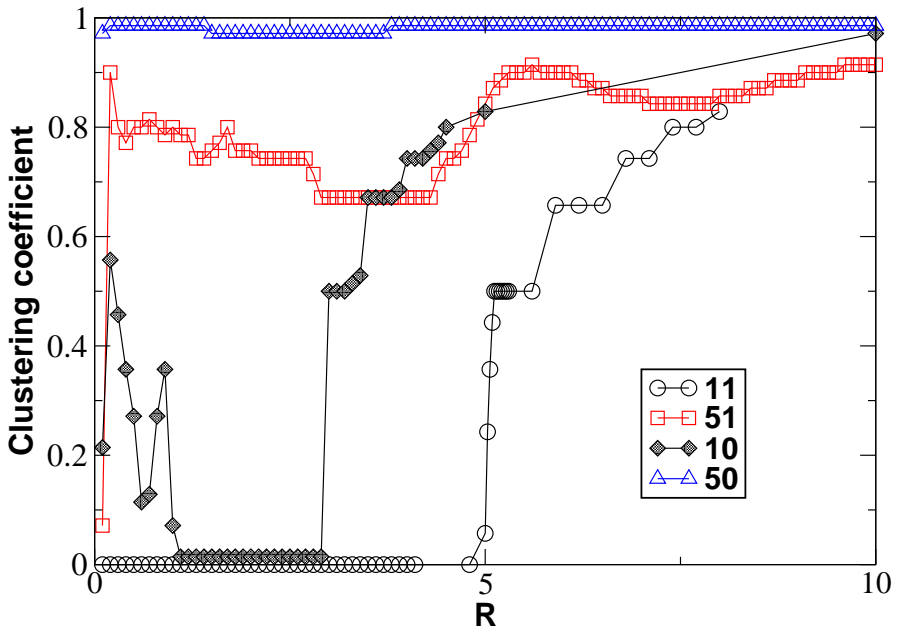

$(1$

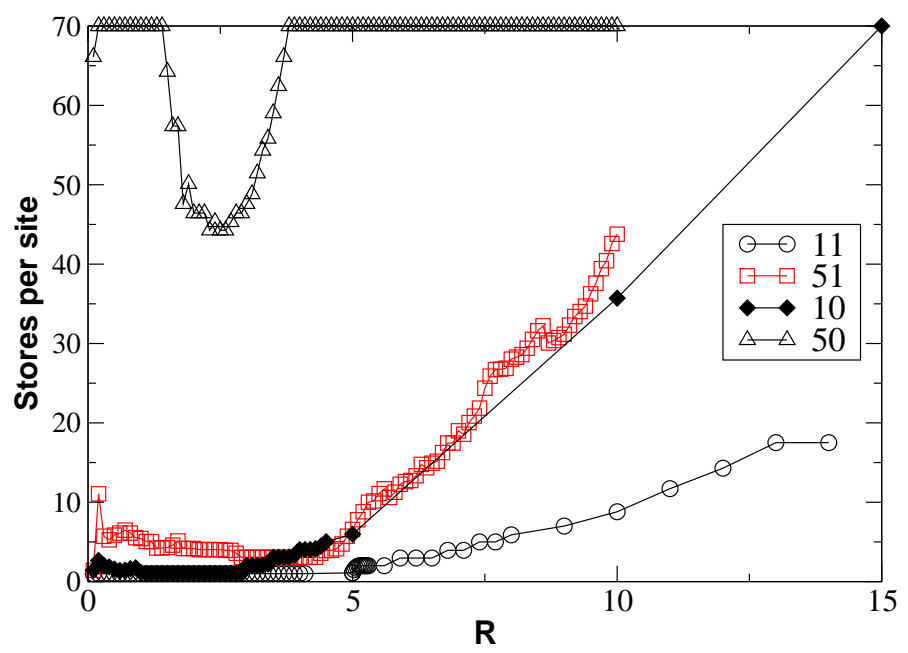

FIG. 8. Clustering coefficient (a) and mean site size (b) obtained for $L=50, T=1, p_{0}=0$, $N_{\text {stores }}=70$ and different values of $Z$ and $\alpha$, shown in that order in the legend box. 


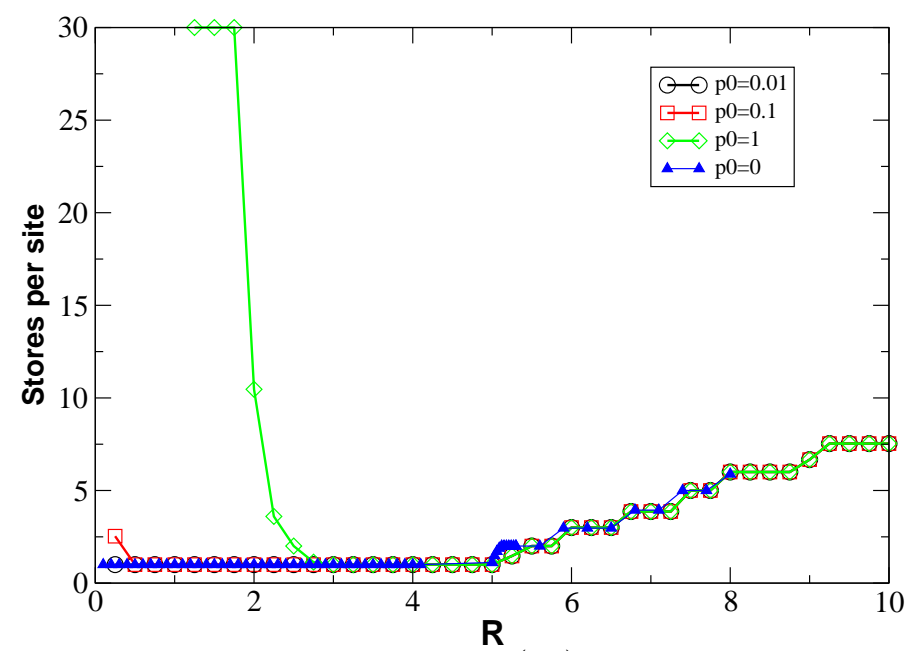

FIG. 9. Effect of the cost price of the product $\left(p_{0}\right)$ on the store aggregation. It is a trivial effect, leading to aggregation for small $R$ values (to allow for some consumers with positive $\mathrm{Ks}$ ) and vanishing as soon as $R$ gets higher than $p_{0}$. All the curves are calculated for $L=50, N_{\text {stores }}=30$, $Z=1$ and $\alpha=1$.

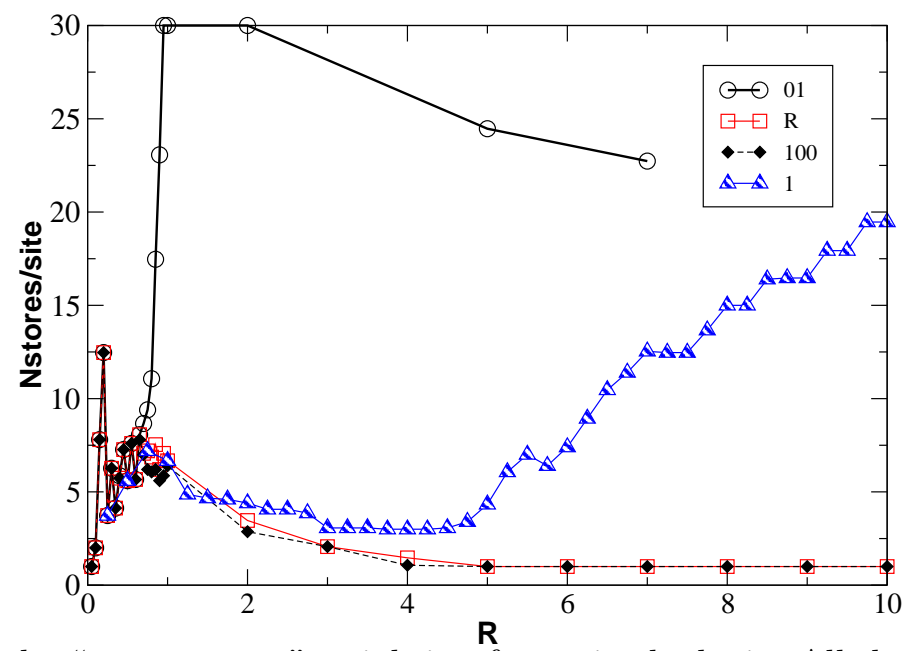

FIG. 10. Effect of the "temperature" weighting factor in the logit. All the curves are calculated for $L=50, N_{\text {stores }}=30, Z=5$ and $\alpha=1$. 


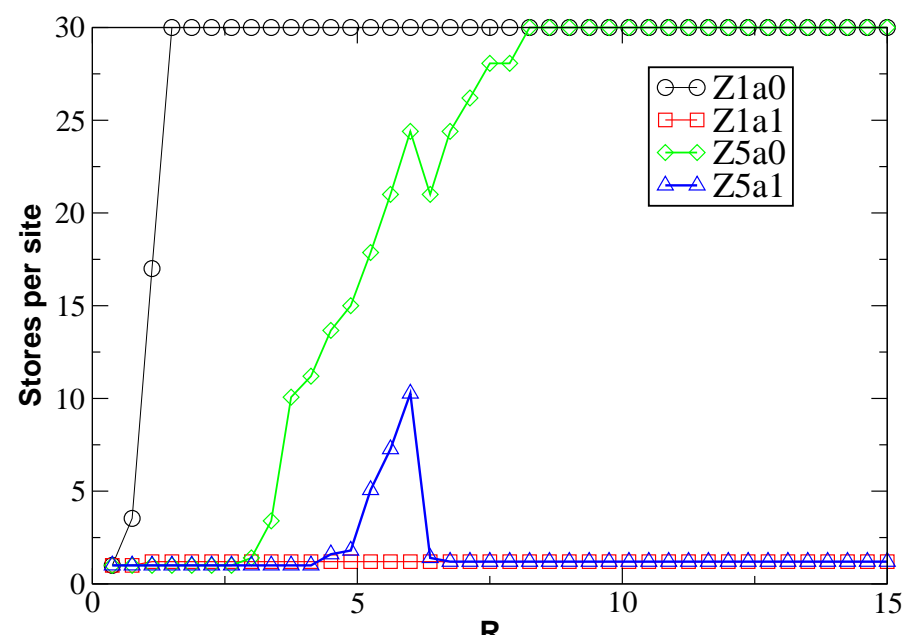

FIG. 11. Effect of a sharp consumer choice. All the curves are calculated for $L=50, p_{0}=0$ and $N_{\text {stores }}=30$. 